\title{
Transmitting diseases by endogenization of 'foreign' immune functional units IFUs spilled over from species with highly similar immune systems
}

\author{
Volker C Hammen* \\ Volker Hammen, Vorstadt 4, 35781 Weilburg, Germany
}

\begin{abstract}
Objective: To identify the potential of cross-species transmissions of genome repeat elements as immune functional units IFUs and their ability to cause diseases in the new host.

Results: The identified principle of innate immunity Pii defined the core functionality of major non-coding repeat elements, for example Endogenous Retroviruses ERVs and transposons, as sequence-based anti-virus registries. An entire ERV encompasses one immune functional unit IFU. In modern immune systems, immune responses appear to be rerouted from IFUs into immune cells. IFUs and immune cells collaborate together to enable immunity against sequence-homolog viruses, immune cells function as readers/controllers of IFUs. The host's immune system controls endogenization of IFUs. Similar immune cells can identify transmitted IFUs from related immune systems as their 'own' IFU and consequently endogenize them.
\end{abstract}

Conclusion: When 'foreign' IFUs are transmitted to new hosts by vectors such as parasites, the new hosts' immune cells endogenize them as their own IFUs into germline cells, causing new diseases in future generations when they are demethylated during ageing.

\section{Introduction}

A great part of animal genomes consists of repeat framed viral genomes, commonly termed non-coding sequences and therefore often called junk-DNA [1]. The functionality of ERVs, transposons and other repeat types is still unknown.

A retrovirus replicates by integration of the viral genome into host cell genomes, and as it is commonly believed in science as well as into host germline cells, where they transmit vertically to the next generation. Around $8 \%$ of the human genome consist of ERVs [2].

The identified principle of innate immunity Pii explains the central functionality of ERVs and transposons as anti-viral sequence registry, and how these registries in collaboration with immune cells in theory guarantee resistance against exogenous viruses with identical or homolog sequences [3].

\section{The concept of ERVs as anti-viral registries acting as a single immune functional unit IFU}

The theory of the principle of innate immunity Pii, which can be described as a natural law, depicts how the host's immune system separates self versus non-self and identifies pathogens on the basis of the pathogen sequence registries. Endogenous virus genomes and other repeats, such as endogenous retroviruses ERVs, exemplarily stand for the host's virus sequence database to immunize the host against viruses and other types of pathogens with similar or homolog sequences. All sequences stored in these anti-viral registry databases are the foundation for innate immunity. It contains sequence information of foreign pathogens. The sequences of detected infecting pathogens are compared with those anti-viral sequence registries stored within the host's genome, namely ERVs and transposons. In case of a positive match, the foreign DNA is inhibited and destructed. According to this theory, ERVs are the sequence fundament utilized and controlled by the host's immune system. It enables immunity against the sequencepositive-matched exogenous viruses.

One complete ERV sequence functions as an independent complete single unit, termed immune functional unit IFU [3] according to its relations to and for the immune system. In cellular immune systems, it has a sequences-comparison-only function, confirmed by methylation of the entire sequence as a fast and easy prevention of IFU transcripts. For this reason, the Pii concept postulates a default methylation [3], neither hyper-methylated nor hypo-methylated, in which all cytosines along the IFU sequence, including the long terminal repeats LTRs, are mono-methylated: the default methylation of IFUs [3]. For this reason, we have to distinguish between genes and IFUs, because genes utilize a variable methylation to regulate gene expression while IFUs should demonstrate a complete sequence-covering default methylation. The common hypo-methylation and hyper-methylation can only refer to genes as IFUs with a partly damaged default methylation still show a higher methylation level than hyper-methylated genes.

*Correspondence to: Volker C Hammen, Volker Hammen, Vorstadt 4, 35781 Weilburg, Germany, E-mail: volkhammen@aol.com

Key words: ERV, transposon, immune functional unit, IFU, spill-over, IFU transmission

Received: January 14, 2019; Accepted: January 25, 2019; Published: January 29,2019 
Immune systems rely on the ability to classify self versus non-self or foreign. The sequence stored in the immune functional unit IFU establishes the main innate classification basis, and in the end is the basis for immunity of the host against sequence-homologue invaders. Furthermore, this immunity can be inherited by en block inheritance of the specific IFU to the next generation.

The repeat frames are identifiers of IFUs, resembling tags indicating to the host's immune system that the sequence within the start repeat and the end repeat is a foreign sequence.

All identified repeat types on the various host genomes are different types of IFU registries, such as endogenous retroviruses ERVs, transposons, further including all common and rare repeat registries as IFUs like short interspersed nuclear elements SINEs, long interspersed nuclear elements LINEs, chicken repeat 1 CR1, Alu repeat elements, intracisternal A-type particle IAP, and added by less abundant repeat types.

\section{The feedback loop for endogenization of IFUs-to-be into germline cells}

The concept of Pii postulates a feedback loop from memory immune cells of the adaptive immune system to germline cells [3]. Similar to IFUs, this feedback loop is mandatorily under the control of the host's immune system and managed most likely by immune cells. This feedback loop is responsible for the de novo endogenization of future IFUs into the genomes of host germline cells. The concept postulates, that the control of endogenization of a new IFU-to-be is a core functionality of the immune system [3] and requires specialized molecules or immune cells to create IFUs in spermatocytes and oocytes under the control of the host's immune system.

\section{IFUs under the control of the host's immune system}

All endogenized ERVs, transposons and other repeat types are originally integrated into the host's genome as IFUs under the control and managed by the host's immune system, as they function for the host's immune system. IFUs are controlled, integrated, managed, utilized, inherited, and deleted by the host's immune system [3]. IFUs are the sequence identification registry collaborating with genes and utilized by the host's immune system, either to enact immune responses directly as in older immune systems like in plants, insects, and early fishes such as lancelets, or the immune response is rerouted into immune cells which have pooled many IFUs and enact a centralized immune response function by immune cells. Exemplarily it was shown how this natural occurring IFU immunization can be expected to be identified as a new virus-registry in the case of the Human Immunodeficiency Virus HIV causing the human disease Acquired Immune Deficiency Syndrome AIDS, which can be expected to be endogenized on survivors' genomes as a new IFU carrying immunization against exogenous HIV [4].

IFUs in the form of ERVs and transposons being controlled by and functioning for the host's immune system is in great contrast to the common scientific thinking that they are genomic parasites [5].

\section{Transmission pathways for cross-species transmission of 'foreign' IFUs}

The view of ERV-IFUs and transposon-IFUs under the control and supervision of the host's immune system gives room for completely new pathways of disease transmission of IFUs, vectors and their impact. By viewing IFUs as registries, with a comparably passive immune response mechanism with regard to the direct immune response from older immune systems, then we require reading cells of the immune system which take over immune responses. According to this interpretation, IFUs are not at all parasitic but under the control of the host's immune system.

The central message is that the immune system of the host is in charge of the IFUs and responsible for the new integration of IFUs-tobe.

The host's immune system enacts their own IFUs as a central mechanism of immunity in collaboration with immune cells. As this immunity system exists in all living beings except viruses, the archetype invented by bacteria and archaea as CRISPR/cas system [6] expanded to full genome IFUs in later evolved organisms, every single host can be expected to assess a foreign new IFU as their own IFU across all species. Yet, when a certain IFU is first endogenized by one species and later spilled over to another species, it still is considered its own IFU by the new host, yet scientist can see that it originates from another immune system and view it as 'foreign' IFU.

Main transmission pathways are either vectors exchanging body components such as tissues or fluids or living in a host-parasite relationship. In either way, cells of one organism are exchanged with another organism including the cell's genomes and their integrated IFUs. With regard to IFUs, the central question is then if the foreign IFU is in the range of the new immune system and can be detected by their immune cells, and further if the new host's immune cells function similar as the original host's immune cells.

Organ transplants can be classified as a modern transmission pathway in which the receiving patient's immune system is able to take up some of the foreign IFUs from the transplanted organ. Pig endogenous retroviruses PERVs were observed to be transmitted to human cells [7]. According to the concept Pii, the human immune system will be capable of taking up these PERVs and integrate them into the human genome of future generations.

\section{Endogenization of 'foreign' IFUs in the new host's genome under the control of the new immune system}

The term 'foreign' IFU depicts that this specific IFU was originally not endogenized by the host's own immune system or its ancestors but by a different host's immune system, likely even from a different species and later transmitted to the new host where it was recognized as IFUregistry and consequently endogenized again.

The functional similarity of the different host's immune systems and their immune cells is decisive if they can utilize a 'foreign' IFU. It appears plausible, that closely related immune systems such as human and primate immune systems can more easily exchange IFUs than distant immune systems like human and plant immune systems.

In all cases, spilled-over 'foreign' IFUs and de novo endogenized in the new species are not an active spill over, yet rather a taking-up by the new immune system once the 'foreign' IFU has entered the new organism and is detected there by closely related immune cells. When immune cells function highly similar as human and primate immune cells, they cannot separate between own and foreign IFU. Therefore, the new host utilizes the spilled-over foreign IFU actively as its own and inherits it in the same way as earlier existing IFUs to future generation.

In contrast to pathogen spill-overs, where the pathogen plays the active role, in IFU spill-overs the new immune system is the active and decisive part in spilling over and utilizing the new IFU. Own and foreign IFUs are integrated as new IFUs on behalf of and under the control of the host's immune system. In difference to conclusions that 
some ERVs are still infectious and this ability to infect is the cause for the spill-over [8], according to the concept of Pii, the transmission to the endogenized virus genome of new hosts is an active process by the new host's immune system.

\section{Diseases caused by recently endogenized ‘foreign' IFUs}

Once a de novo IFU is endogenized into the host's genome, it can cause diseases as soon as the methylation protection, designed as default methylation to inhibit IFU transcriptions, starts coding. As the default methylation can be damaged by a wide range of factors of internal or external source, after some time and damage events, shorter IFU sequences start coding which resembles a natural process for all IFUs.

Two exemplary cases for a foreign IFU insertion are the koala retrovirus KoRV [9] and the HERV-K (HML-2).

The Koala retrovirus KoRV often demonstrates intact and infectious open reading frames ORFs, indicating a recent spill-over, an ongoing endogenization $[9,10]$ and population wide spread of KoRV within the population of the koala Phascolarctos cinereus. The KoRV is a decent show case for illustrating that a transmitted spilled-over foreign IFU is taken up by a new host with a similar immune system and de novo endogenized and causing a disease in the new host. The gibbon ape leukemia virus GaLV is highly sequence homolog to the KoRV though their habitats are not overlapping [11]. Several species were identified with similar ERV sequences among them a bat species with population areas overlapping both gibbon and koala habitats [11], which explains how distant species can be infected with the same foreign IFU. An analysis places the Rhinolophus ferrumequinum retrovirus RfRV of the greater horseshoe bat species Rhinolophus ferrumequinum at the root for both, the GaLV and the KoRV [8]. The Koala retrovirus KoRV resembles a new, recent and ongoing population-wide IFU endogenization later causing lymphoma and leukemia in koalas, although most of the circa 25 endogenous KoRVs are defect [12] in an underpinning behaviour of the new host's immune system to deactivate the oncogenic sequences. Albeit deletion of the new IFU does not appear to be an option for the new host's immune system, here the Koala immune system. The history of KoRV-IFU population-wide endogenization can be traced back more than 120 years [13].

A human case of transmitted IFU diseases is demonstrated by HERV-K / HML-2, an example in humans for a recent spilled-over foreign IFU which is being integrated into the human genome. As many as 113 HERV-K / HML-2 were found by the year 2005 on the human genome [14]. HERV-K10 was related with schizophrenia-associated and bipolar-disorder-associated samples [15]. Increased expression levels of HERV-W in blood cells were observed in first episode schizophrenia patients [16]. Sequence differences in HERV-W env sequences were identified from patients with schizophrenia compared to bipolar disorder [17]. Nevertheless, most HERVs have been associated with human cancers such as prostate cancer [18] and breast cancer [19]. Higher numbers of HML-2 K113 insertions observed in African humans compared to the general human population [20] illustrate a still ongoing foreign IFU transmission accompanied by further pathogenic pressures in African populations after the time when human ancestors separated.

\section{Immunity is highest priority for the host}

It becomes apparent that the endogenization of IFUs to convey and ensure immunity of the host and future generations has a much higher priority for the host than the long-term disease impacts in long-lived individuals. The rational can be described by an evolutionary rule that surviving infections is more important than preventing diseases that may develop 30 years after the infection had occurred.

Nevertheless, during the life cycle of the IFU, the hosts target to minimize long term disease effects by mutating IFUs as observed with some of the transmitted foreign KoRV IFUs [12], which were defect within a few generations to prevent cancer development.

\section{Examples of IFU transmissions}

IFU transmissions were identified between host and parasitic species by blood sucking vectors [21]. Several cases of foreign IFU transmissions were reported, in which parasites were suspected as being the main vector for horizontal transmission of the Tc1 repeat, which teleost fishes apparently received from their parasite lampreys [22]. Potential horizontal transmissions of retrotransposons as suspected foreign IFUs were observed between birds and humans via pathogenic insect-borne nematodes [23] thus transferring IFUs to humans which originated from birds. Several cross-species transmissions of endogenous retroviruses ERV-IFUs on an intercontinental scale were investigated, involving more than eleven mammalian orders [24]. These transmissions involved some apparently distant species such as primates and dolphins, spanning several ecosystems, and are still visible in 26 identified species-transmission events [24].

\section{Conclusion}

\section{The IFU transmission is a new pathway of disease transmission}

From all these incidences and based on the concept of immune functional units IFUs being controlled by the host's immune system, it appears feasible to view these circumstances as a pathway of disease transmission. Albeit it seems to be a new pathway in science, it is an ancient process in nature.

\section{Main limitations}

The central limitation of the concept of Pii regarding IFUs is the lack of a proof in an animal model before and after endogenization of an IFU and its capability to convey immunity.

Research needs to re-focus on the possibility that immune systems play active roles in the endogenization of IFUs, and furthermore, in transmission of IFUs from other species.

\section{References}

1. Pennisi E (2012) Genomics. ENCODE project writes eulogy for junk DNA. Science 337: 1159-1161. [Crossref]

2. Gifford R, Tristem M (2003) The evolution, distribution and diversity of endogenous retroviruses. Virus Genes 26: 291-315. [Crossref]

3. Hammen VC (2018) Ars Immunitas: The source and fate of innate immunity. The principle of innate immunity Pii, the concept of the natural law of genomic immune functional units as antiviral registries, the three major pathways of disease, and the 4th pathway of ageing epubli, Munchen, Weilburg, Germany, 580.

4. Hammen VC (2018) The concept of immune functional units for ERVs and transposons and how the natural occurring immunization on the human immune system would look like for HIV. Journal of Translational Science 5: 1-3.

5. Eisenberg E (2016) Proteome diversification by genomic parasites. Genome Biol 7: 17. [Crossref]

6. Garneau JE, Dupuis ME, Villion M, Romero DA, Barrangou R, et al. (2010) The CRISPR/Cas bacterial immune system cleaves bacteriophage and plasmid DNA. Nature 468: 67-71.

7. Patience C, Takeuchi Y, Weiss RA (1997) Infection of human cells by an endogenous retrovirus of pigs. Nat Med 3: 282-286. [Crossref] 
8. Cui J, Tachedjian M, Wang L, Tachedjian G, Wang LF, et al. (2012) Discovery of retroviral homologs in bats: Implications for the origin of mammalian gammaretroviruses. Journal of Virology 86: 4288-4293.

9. Tarlinton RE, Meers J, Young PR (2006) Retroviral invasion of the koala genome. Nature 442: 79-81. [Crossref]

10. Tarlinton R, Meers J, Young P (2008) Biology and evolution of the endogenous koala retrovirus. Cell Mol Life Sci 65: 3413-3421. [Crossref]

11. Denner J (2016) Transspecies Transmission of gammaretroviruses and the origin of the gibbon ape leukaemia virus (GaLV) and the koala retrovirus (KoRV). Viruses 8: 336.

12. Hanger JJ, Bromham LD, McKee JJ, O’Brien TM, Robinson WF (2000) The nucleotide sequence of Koala (Phascolarctos cinereus) retrovirus: A novel type $\mathrm{c}$ endogenous virus related to gibbon ape leukemia virus. Journal of Virology 74: 4264-4272.

13. Ávila-Arcos MC, Ho SYW, Ishida Y, Nikolaidis N, Tsangaras K, et al. (2013) One hundred twenty years of koala retrovirus evolution determined from museum skins. Molecular Biology and Evolution 30: 299-304.

14. Belshaw R, Dawson ALA, Woolven-Allen J, Redding J, Burt A, et al. (2005) Genomewide screening reveals high levels of insertional polymorphism in the human endogenous retrovirus family HERV-K(HML2): Implications for present-day activity. Journal of Virology 79: 12507-12514.

15. Frank O, Giehl M, Zheng C, Hehlmann R, Leib-Mosch C, et al. (2005) Human endogenous retrovirus expression profiles in samples from brains of patients with schizophrenia and bipolar disorders. Journal of Virology 79: 10890-10901.

16. Yao Y, Schroder J, Nellaker C, Bottmer C, Bachmann S, et al. (2008) Elevated levels of human endogenous retrovirus-W transcripts in blood cells from patients with first episode schizophrenia. Genes, Brain and Behavior 7: 103-112.
17. Perron H, Hamdani N, Faucard R, Lajnef M, Jamain S, et al. (2012) Molecular characteristics of Human Endogenous Retrovirus type-W in schizophrenia and bipolar disorder. Transl Psychiatry 2: e201.

18. Ishida T, Obata Y, Ohara N, Matsushita H, Sato S, et al. (2008) Identification of the HERV-K gag antigen in prostate cancer by SEREX using autologous patient serum and its immunogenicity. Cancer Immunity Archive 8: 1-15.

19. Wang-Johanning F, Frost AR, Jian B, Epp L, Lu DW, et al. (2003) Quantitation of HERV-K env gene expression and splicing in human breast cancer. Oncogene 22: 15281535. [Crossref]

20. Wildschutte JH, Williams ZH, Montesion M, Subramanian RP, Kidd JM, et al. (2016) Discovery of unfixed endogenous retrovirus insertions in diverse human populations. Proceedings of the National Academy of Sciences 113: E2326-E2334.

21. Gilbert C, Schaack S, Pace Ii JK, Brindley PJ, Feschotte C (2010) A role for hostparasite interactions in the horizontal transfer of transposons across phyla. Nature 464 : $1347-1350$.

22. Kuraku S, Qiu H, Meyer A (2012) Horizontal Transfers of Tc1 Elements between teleost fishes and their vertebrate parasites, lampreys. Genome Biology and Evolution 4: 929-936

23. Suh A, Witt CC, Menger J, Sadanandan KR, Podsiadlowski L, et al. (2016) Ancient horizontal transfers of retrotransposons between birds and ancestors of human pathogenic nematodes. Nature Communications 7: 11396.

24. Diehl WE, Patel N, Halm K, Johnson WE (2016) Tracking interspecies transmission and long-term evolution of an ancient retrovirus using the genomes of modern mammals. Life 5: e12704.

Copyright: $@ 2019$ Hammen VC. This is an open-access article distributed under the terms of the Creative Commons Attribution License, which permits unrestricted use, distribution, and reproduction in any medium, provided the original author and source are credited. 